\title{
AI and data-driven Christian Theology
}

Ben Geoffrey A S

Independent Researcher

ORCID : 0000-0001-6545-6314

Correspondence : bengeof@gmail.com

\begin{abstract}
An NLP based AI tool was developed for topic mining Christian writings of major figures from different Christian eras and movements. The text corpus chosen for text mining includes : the volumes of Augustine and John Chrysostom from the patristic period, the writings of Thomas Aquinas from the Scholastic period, the writings of John Calvin the reformer and the text corpus of sermons by George Whitefield and John Wesley that mark the beginning of the modern Christian evangelical movement and evangelicalism. The topics text mined and topics summarized include : Gospel, Salvation, Jesus Christ, Sin, Temptation, Tribulation, Pride, Lust, Envy, Joy, Hope, Charity, Marriage, Church, Heaven, Hell. They are downloadable from the link https://drive.google.com/drive/folders/1tG2Y36MF1ApvYnhPM njtNvKYiv7 8RL?usp=sharing and this is expected to help Christian content developers. The link to the code of the NLP tool hosted in the GitHub repository is provided to aid further development https://github.com/bengeof/AI-driven-Theology-Open-Development. These tools must not be viewed as threatening the role of the human in humanities research broadly and specifically in Christian theology but as empowering the human with additional powers of AI for research in the humanities in the age of Big Data.
\end{abstract}




\section{Introduction}

Natural language processing can be defined as a means by which computers can recognize and process human language in the form of text or speech [1]. Many successful attempts of Natural Language Processing has been in indexing of web pages by search engines, performing sentiment analysis of product reviews on e-commerce sites, opinion mining social media and attempts to use NLP based automated surveillance intelligence generation to counter terrorism [2-10]. Search engines run NLP algorithms on web pages through a process called crawling and index web pages with associated keywords so as to enable them to respond to user search queries based on keywords [11-13]. Sentiment analysis NLP techniques have been used to automate the process of accessing product reviews and comments on e-commerce sites [14,15]. NLP based chatbots have also been developed in an attempt to provide automated customer support in e-commerce and the like industries [16]. NLP based text or speech labelling, tagging and classification by topics has also been developed for the programmatically automated generation of surveillance intelligence which have been of interest for security purposes $[10,17,18]$. In the present work we propose an NLP algorithmic workflow that attempts to bring the power of Artificial Intelligence and computers to research in humanities and to Christian Theology in particular. Artificial Intelligence becomes significantly useful in any area where processing Big Data is required and statistical learning can be employed on Big Data libraries to generate insights from the data. This provides significant aid to human intelligence in the processing of Big Data.

The advent of the Internet and cheap information access to huge data publicly accessible data brings with it a set of opportunities as well as challenges. For those among the humanities research community there is huge opportunity to the access to writings of historians, philosophers, theologians, thinkers, poets, writers and artists across centuries through initiatives such as wikibooks, wikisource and other such text libraries that host public domain literature for public access [19]. However the challenge is the human limitation on the amount of literature one can plough through when researching on any particular topic considering the vastness of literature available right now. Therefore to assist our efforts in this, we propose a NLP based programmatic tool that is programmatically automated to text mine large library of raw text data and extract only relevant text pertinent to research topic of the researcher by the keywords the characterize the research topic which the program takes as input from the user. After this process of automatically collecting text data that is relevant to the research topic from a large library of text data, the program attempts to summarize in the best possible way all relevant information available in the text related to the research topic. 
These tools must not be viewed as threatening the role of the human in humanities research but as empowering the human with additional powers of AI for research in the humanities in the age of Big Data.

\section{Methods}

The algorithmic workflow of the programmatic tool is explained below with the help of a block diagrammatic representation of the algorithm. The text corpus to be mined was obtained from the Online Christian Classics Ethereal Library[20]. The library offers the download of an exhaustive list of Christian literature in the public domain in XML format which is preferred by text mining as it offers text data in a semi-structured format. A selection of Christian writings of major figures from different Christian eras and movements were selected for text mining. The corpus chosen for text mining includes : the volumes of Augustine and John Chrysostom from the patristic period, the writings of Thomas Aquinas from the Scholastic period, the writings of John Calvin the reformer and the text corpus of sermons by George Whitefield and John Wesley that mark the begining of the modern Christian evangelical movement and evangelicalism. The text corpus was cleaned by way of removing Title, Preface, Index, Header, Footer, Notes, Endnotes and Page Number XML tags

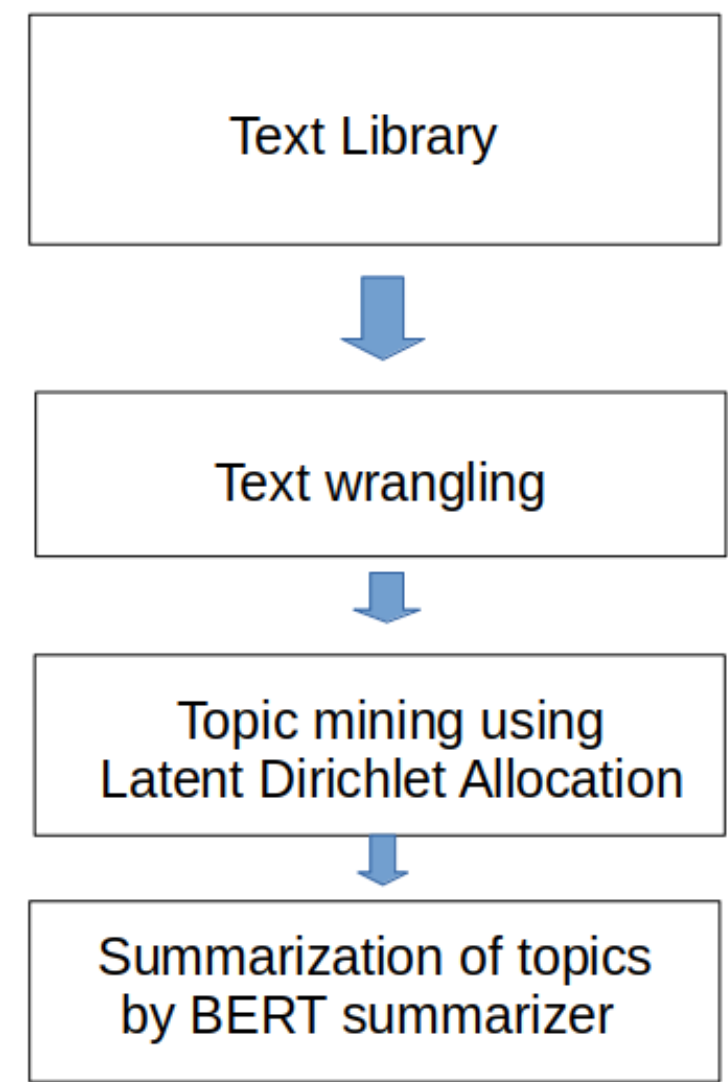

Fig. 1 Block diagrammatic workflow of the algorithmic flow of the programmatic tool. 
On the cleaned text, Latent Dirichlet Allocation(LDA) method was applied to identify key topics of the text [21]. The text corresponding to the key topics identified by the LDA method was extracted and summarized using the BERT summarizer [22]. Topics of general interest to Chrisitan content developers were also text mined from the text and summarized. The summarizes can be downloaded from the link below

https://drive.google.com/drive/folders/1tG2Y36MF1ApvYnhPM njtNvKYiv7 8RL?usp=sharing

The Jupyter Notebook (code) used is available in the GitHub repository below for further future development

https://github.com/bengeof/AI-driven-Theology-Open-Development

\section{Results and Discussion}

The topics text mined and topics summarized include : Gospel, Salvation, Jesus Christ, Sin, Temptation, Tribulation, Pride, Lust, Envy, Joy, Hope, Charity, Marriage, Church, Heaven, Hell. The key topics identified by the LDA in individual writers were in agreement with general humanly carried out charaterization of individual writers. For example, The LDA identified the evangelicalism of the evangelical preachers George Whitefield and John Wesley in contrast to the intellectualism of Thomas Aquinas as shown in Fig. 2, 3 \& 4. The summaries generated by the BERT summarizer on the key topics were cogent, meaningful and can be used by Christian content developers of research and homiletic interest. Thus our tool introduces AI and Big Data driven Christian content development. The AI does not threaten the role of humans in Chrisitan content development but serves as a technological aid in the era of Big Data in content development. 
Jupyter Notebook for Thomas Aquinas Last Checkpoint: a few seconds ago (autosaved)

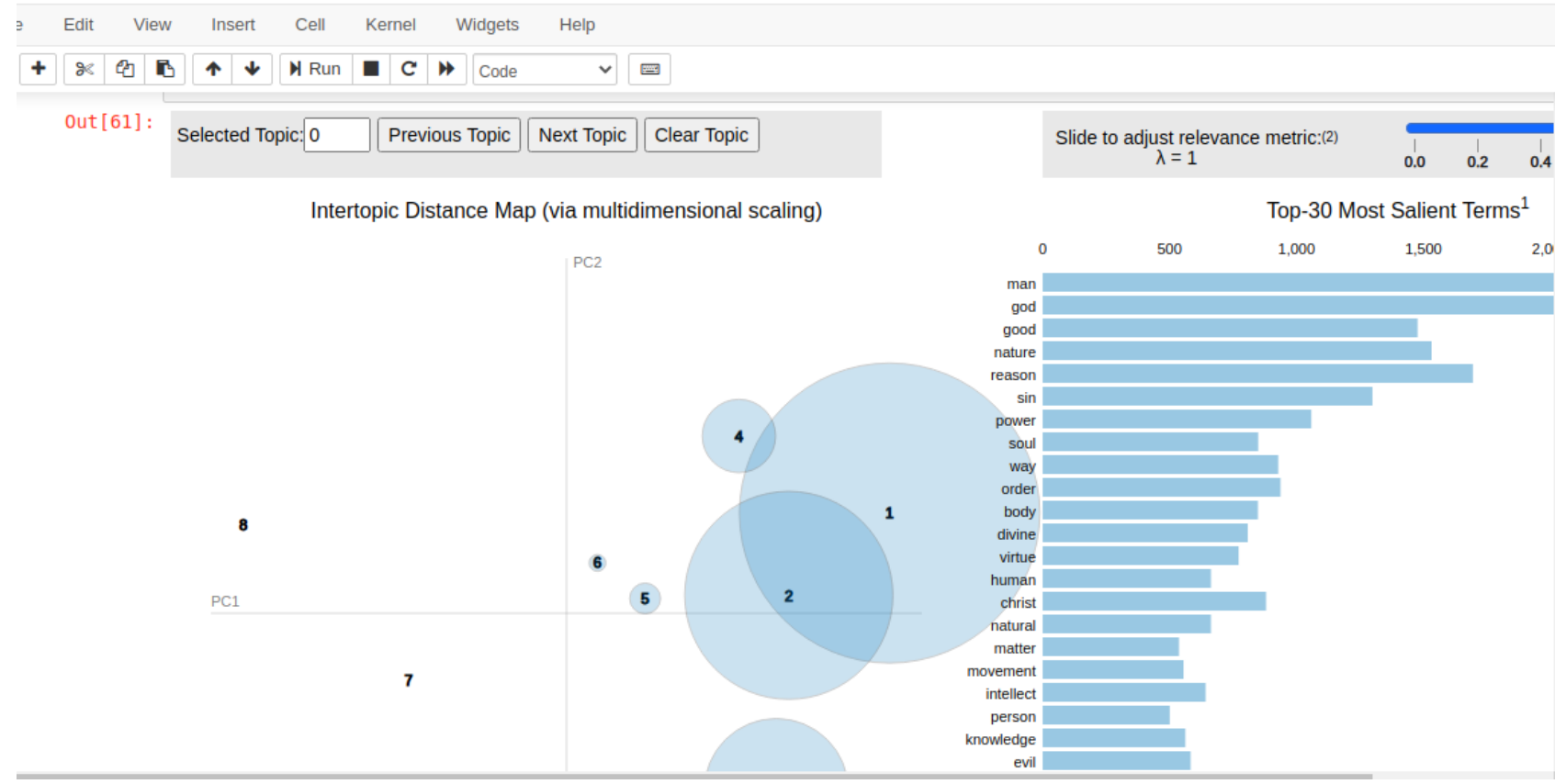

Fig. 2 - LDA topic mining of Thomas Aquinas

'- jupyter Notebook for John Wesley Last Checkpoint: a few seconds ago (autosaved)

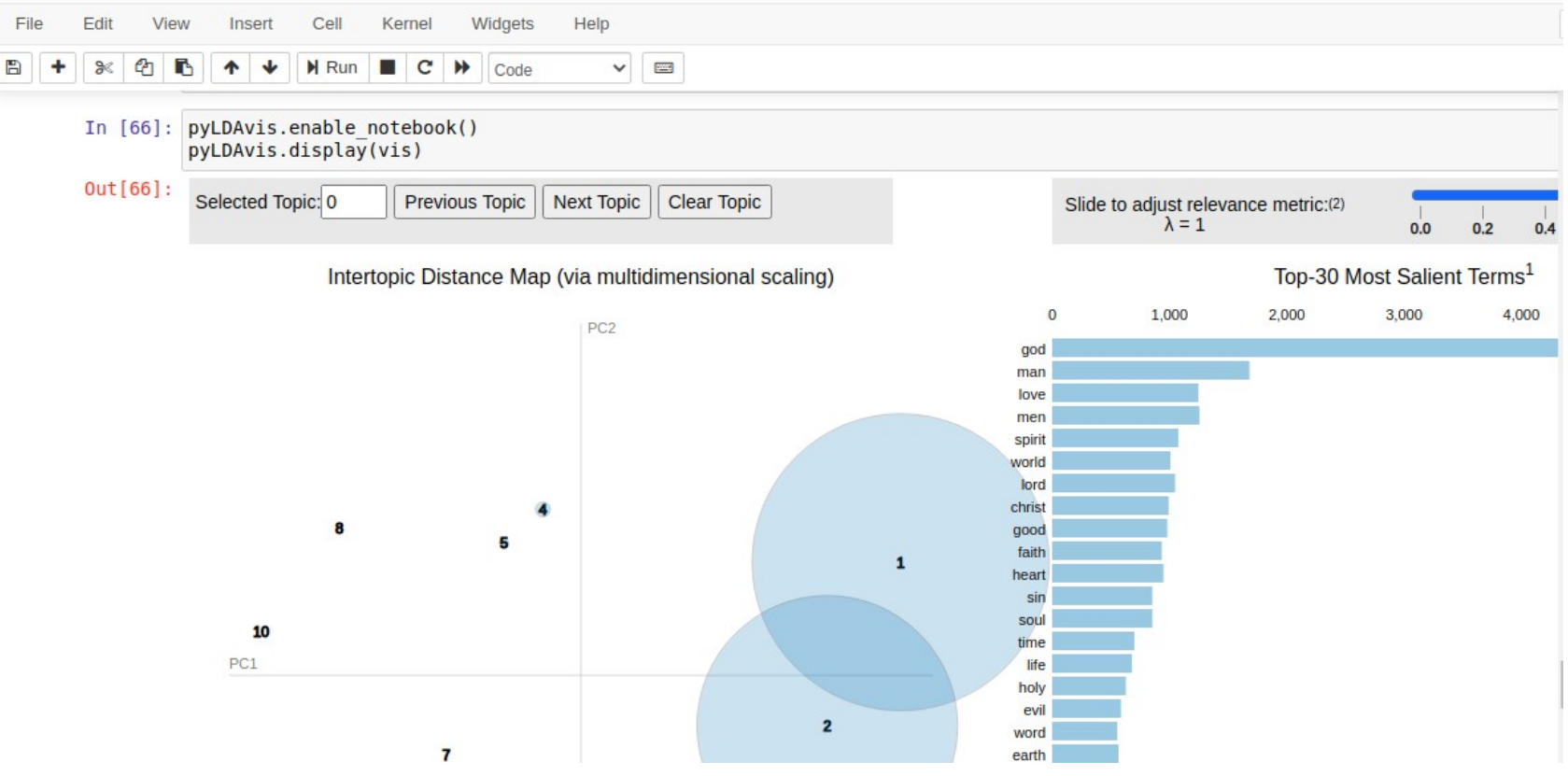

Fig. 3 - LDA topic mining of John Wesley 


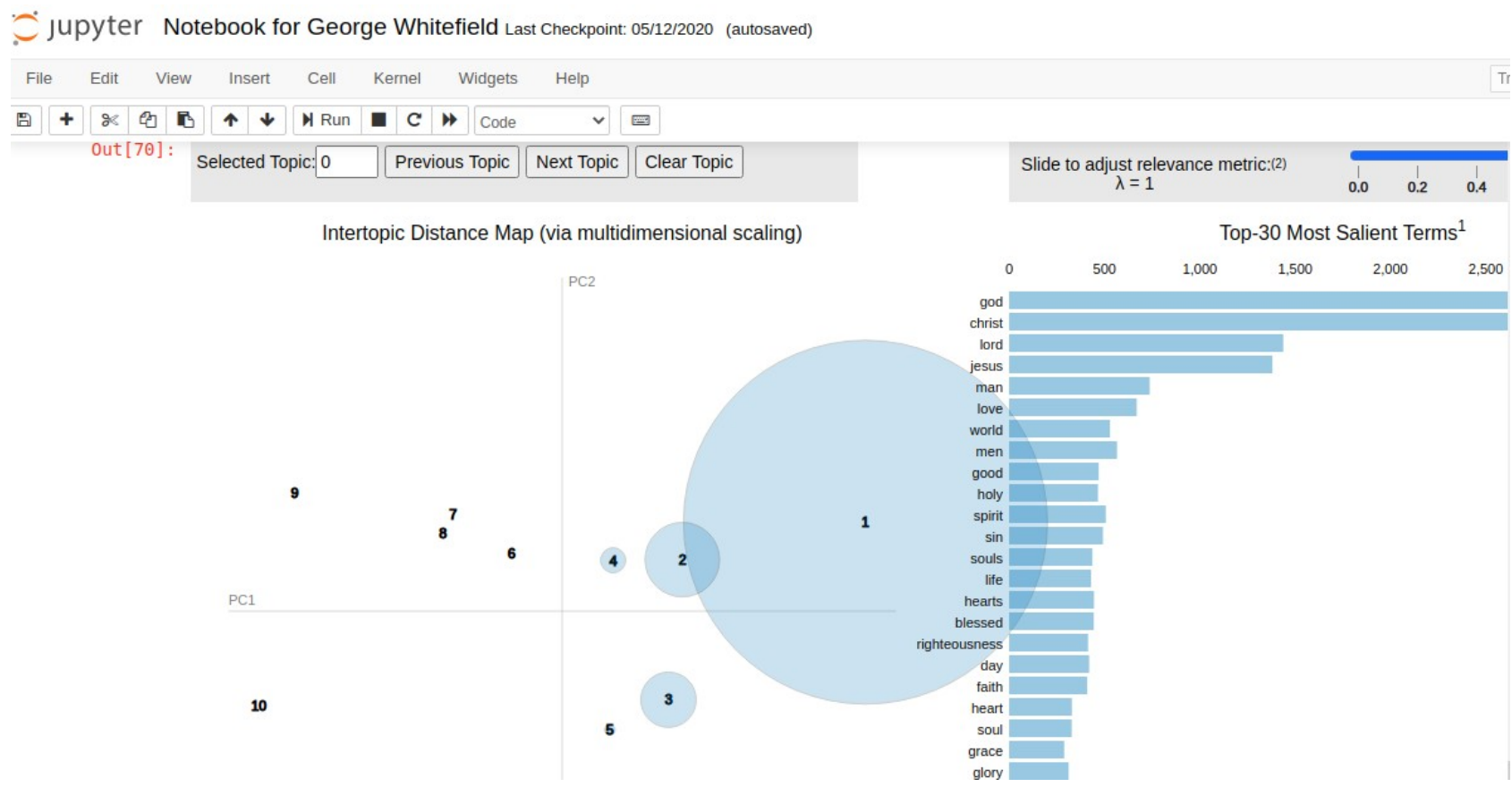

Fig. 4 - LDA topic mining of George Whitefield

\section{Conclusion and future outlook}

An NLP based AI tool was developed for topic mining Christian writings of major figures from different Christian eras and movements. The text corpus chosen for text mining includes : the volumes of Augustine and John Chrysostom from the patristic period, the writings of Thomas Aquinas from the Scholastic period, the writings of John Calvin the reformer and the text corpus of sermons by George Whitefield and John Wesley that mark the beginning of the modern Christian evangelical movement and evangelicalism. The topics text mined and topics summarized include : Gospel, Salvation, Jesus Christ, Sin, Temptation, Tribulation, Pride, Lust, Envy, Joy, Hope, Charity, Marriage, Church, Heaven, Hell. They are downloadable from the link https://drive.google.com/drive/folders/1tG2Y36MF1ApvYnhPM njtNvKYiv7 8RL?usp=sharing and this is expected to help Christian content developers. The link to the code of the NLP tool hosted in the GitHub repository is provided to aid further development https://github.com/bengeof/AI-driven-Theology-Open-Development. These tools must not be viewed as threatening the role of the human in humanities research broadly and specifically in Christian theology but as empowering the human with additional powers of AI for research in the humanities in the age of Big Data. 


\section{References}

1. Chowdhary, K. R. "Natural language processing." Fundamentals of Artificial Intelligence. Springer, New Delhi, 2020. 603-649.

2. Voorhees, Ellen M. "Natural language processing and information retrieval." International summer school on information extraction. Springer, Berlin, Heidelberg, 1999.

3. Ferrari, Alessio, Beatrice Donati, and Stefania Gnesi. "Detecting domain-specific ambiguities: an NLP approach based on Wikipedia crawling and word embeddings." 2017 IEEE 25th International Requirements Engineering Conference Workshops (REW). IEEE, 2017.

4. Rajput, Adil. "Natural Language Processing, Sentiment Analysis, and Clinical Analytics." Innovation in Health Informatics. Academic Press, 2020. 79-97.

5. Tanguy, Ludovic, et al. "Natural language processing for aviation safety reports: from classification to interactive analysis." Computers in Industry 78 (2016): 80-95.

6. Sun, Shiliang, Chen Luo, and Junyu Chen. "A review of natural language processing techniques for opinion mining systems." Information fusion 36 (2017): 10-25.

7. Maynard, Diana, and Adam Funk. "Automatic detection of political opinions in tweets." Extended Semantic Web Conference. Springer, Berlin, Heidelberg, 2011.8. Marrese-Taylor, Edison, Juan D. Velásquez, and Felipe Bravo-Marquez. "A novel deterministic approach for aspectbased opinion mining in tourismproducts reviews." Expert Systems with Applications 41.17 (2014): 7764-7775.

9. Sun, Shiliang, Chen Luo, and Junyu Chen. "A review of natural language processing techniques for opinion mining systems." Information fusion 36 (2017): 10-25.

10. Peter Margulies, Surveillance By Algorithm: The NSA, Computerized Intelligence Collection, and Human Rights, 68 Fla. L. Rev. 1045 (2016)

11. Nesi, Paolo, Gianni Pantaleo, and Gianmarco Sanesi. "A Distributed Framework for NLPBased Keyword and Keyphrase Extraction From Web Pages and Documents." DMS. 2015. 12. Krapivin, Mikalai, et al. "Keyphrases extraction from scientific documents: improving machine learning approaches with natural language processing." International Conference on Asian Digital Libraries. Springer, Berlin, Heidelberg, 2010.

13. Armentano, Marcelo G., et al. "NLP-based faceted search: Experience in the development of a science and technology search engine." Expert systems with applications 41.6 (2014): 2886-2896. 14. Fang, Xing, and Justin Zhan. "Sentiment analysis using product review data." Journal of Big Data 2.1 (2015): 5.

15. Shivaprasad, T. K., and Jyothi Shetty. "Sentiment analysis of product reviews: a review." 2017 International Conference on Inventive Communication and Computational 
Technologies (ICICCT). IEEE, 2017.

16. Handoyo, Eko, et al. "Ticketing Chatbot Service using Serverless NLP Technology." 2018

5th International Conference on Information Technology, Computer, and Electrical

Engineering (ICITACEE). IEEE, 2018.

17. Wiil, Uffe Kock, Nasrullah Memon, and Jolanta Gniadek. "Crimefighter: A toolbox for counterterrorism." International Joint Conference on Knowledge Discovery, Knowledge

Engineering, and Knowledge Management. Springer, Berlin, Heidelberg, 2009.

18. Ball, Leslie. "Automating social network analysis: A power tool for counter-terrorism." Security Journal 29.2 (2016): 147-168.

19. Armstrong, Timothy K. "Rich texts: Wikisource as an open access repository for law and the humanities." U of Cincinnati Public Law Research Paper 10-09 (2010).

20. Kooy, Brian K. "Christian Classics Ethereal Library." Reference Reviews (2011).

21. Tong, Zhou, and Haiyi Zhang. "A text mining research based on LDA topic modelling." In International Conference on Computer Science, Engineering and Information Technology, pp. 201210. 2016.

22. Miller, Derek. "Leveraging BERT for extractive text summarization on lectures." arXiv preprint arXiv:1906.04165 (2019). 